\title{
Pediatric Discoid Lupus Erythematosus: Successful Treatment with Antimalarials
}

\author{
Poonam Adlakha $^{1 *}$, Mohit Nagpal ${ }^{2}$, Rahul Mahajan ${ }^{2}$ and Sanjeev Handa ${ }^{2}$ \\ ${ }^{1}$ Department of Histopathology, Postgraduate Institute of Medical Education and Research, Chandigarh, India \\ ${ }^{2}$ Department of Dermatology, Venereology, and Leprology, Postgraduate Institute of Medical Education and Research, Chandigarh, India
}

\begin{abstract}
Pediatric discoid lupus erythematous (DLE) is an uncommon entity that clinically resembles the adult onset DLE. However, pediatric DLE shows a higher incidence of positive family history and higher propensity towards progression to systemic lupus erythematous. Herein, we report a case of pediatric DLE who responded well to oral hydroxychloroquine.
\end{abstract}

Keywords: Discoid Lupus Erythematous, Systemic Lupus Erythematous, Pediatric

\section{Introduction}

Discoid lupus erythematous (DLE) belongs to the category of lupus erythematous (LE), a group of disorders that are linked together by the development of autoimmunity against the molecular constituents of nucleosomes and ribonucleoproteins. It is not common in childhood and rare in infancy. This is consistent with the observation that the overall incidence of connective tissue diseases is much lower in children than adults. ${ }^{[1]}$ In addition; the clinical picture is more variable as mucocutaneous manifestations develop frequently during the varying course of disease. We report a case of pediatric DLE who responded well to oral hydroxychloroquine.

\section{Case Report}

A 12-years old female presented to the dermatology outpatient with complaints of scaly, erythematous plaques on head and neck of 3 years duration. The lesions began as an asymptomatic red papule over the bridge of the nose which increased in size to form a plaque. Over the next 8- 10 months, similar lesions appeared on cheeks, external ears, scalp, and neck. The plaques over scalp resulted in scarring alopecia. Although the child complained of photosensitivity, there were no associated systemic complaints. Similar lesions were present in father for last 1012 years, which had responded partially to the application of topical clobetasol propionate ointment. On examination, multiple well defined discoid erythematous plaques, varying in size from 0.5 to $3 \mathrm{~cm}$ in diameter with adherent scales and central atrophy were present over the bridge of nose and both malar areas (Figure 1). Mucosa and nails were normal. Histopathologic examination of skin biopsy from one of the plaques showed epidermal hyperkeratosis and focal hypergranulosis. Dermis showed edema and myxoid change with moderate to heavy peri-appendageal and perivascular nodular lymphocytic infiltrate, occasional follicular plugging, melanin incontinence and focal basal cell vacuolation (Figure 2 and 3). PAS and Alcian blue staining highlighted mucinous degeneration (Figure 4). Direct immunofluorescense (from non-exposed lesional skin) showed granular deposition of $\operatorname{IgG}(++)$ with patchy deposits of $\operatorname{IgM}(+)$ and $\mathrm{C} 3(+)$ along the basement membrane zone (Figure 5). Complete blood counts, serum biochemistry, routine urinalysis, 24-hour urine proteins and serum complement levels were with-in normal limits. Immunologic investigations like ANA, anti-ds DNA, antiRo, anti-La and complement levels were negative. The child was advised strict photoprotection (sun avoidance and broad spectrum sun screen of SPF30) and topical clobetasol propionate ointment. After an ophthalmology clearance, she was started on oral hydroxchloroquine 150 $\mathrm{mg} /$ day $(5 \mathrm{mg} / \mathrm{kg} / \mathrm{d})$ with which the lesions subsided after 2 months of treatment.

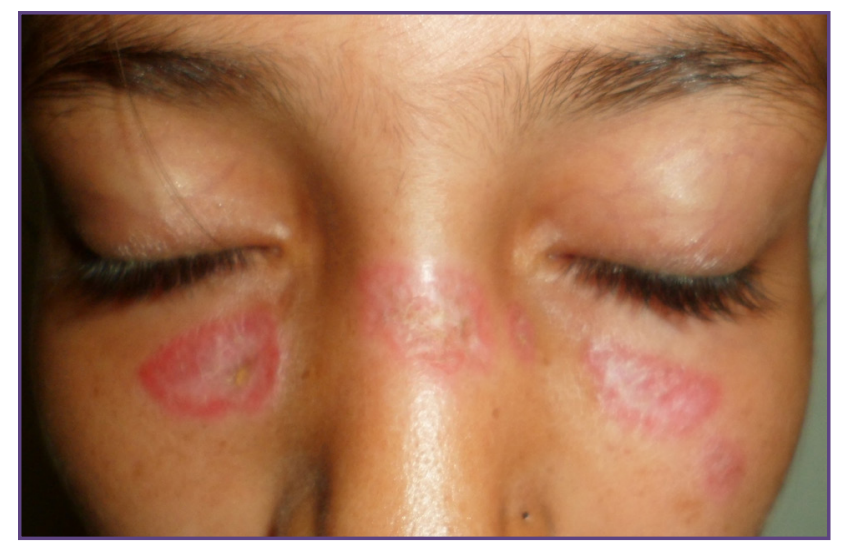

Fig. 1: Multiple discoid erythematous plaques with adherent scales and central atrophy present over the bridge of nose and malar areas. 


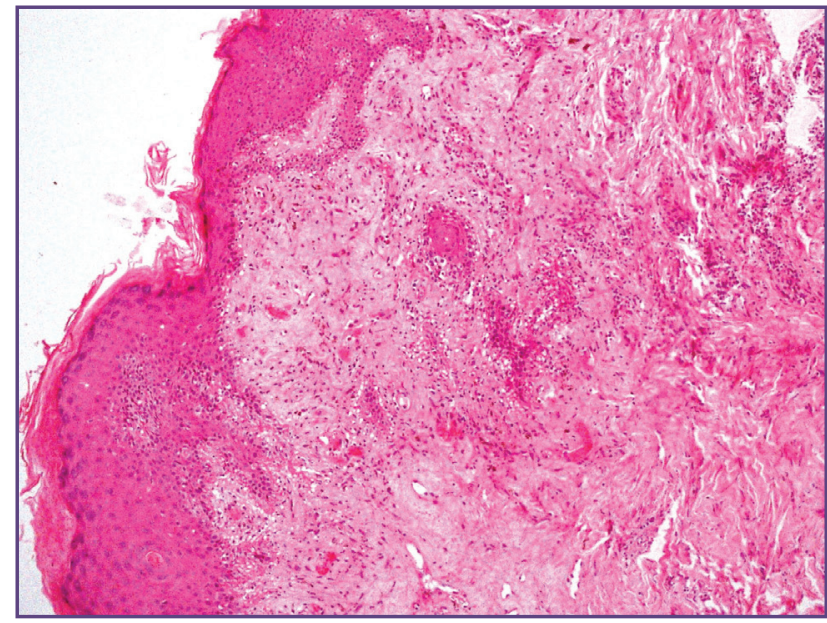

Fig. 2: Skin biopsy shows mild hyperkeratosis, focal follicular plugging and irregular acanthosis of the epidermis. The dermis shows mild to moderate lymphomononuclear infiltrate predominantly along the dermoepidermal junction extending upwards causing basal cell degeneration. (H/E, x 200).

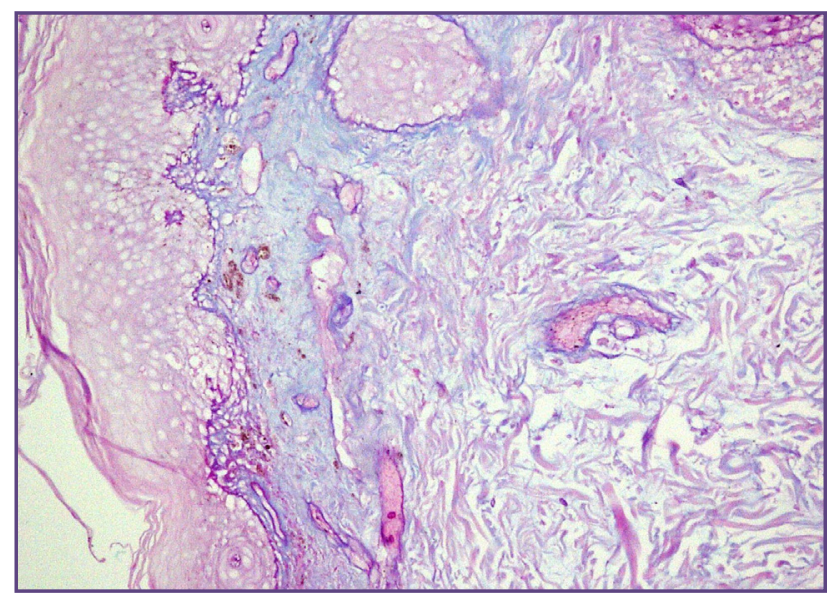

Fig. 4: Mucinous degeneration seen on PAS-Alcian blue staining (x200).

\section{Discussion}

Less than $5 \%$ of patients with DLE develop the disease before they reach the age of 16 and less than $2 \%$ of patients with DLE have an onset before 10 years of age. A female predominance has been observed in some studies in childhood DLE (Moises-Alfaro et al - 5:1) but not in others (Sampaio et al - 2:1). ${ }^{[2,3]}$ As in adults, the exact etiology of childhood DLE remains obscure. Reports of familial cases and a high concordance for clinical disease in monozygotic twins suggest a strong genetic component and altered cellular immunity in genetically predisposed individuals as factors in its pathogenesis. In a study, family history of LE was present in 4 of $34(11.8 \%)$ patients compared to $1 \%$ $4.4 \%$ in adult DLE. ${ }^{[3]}$

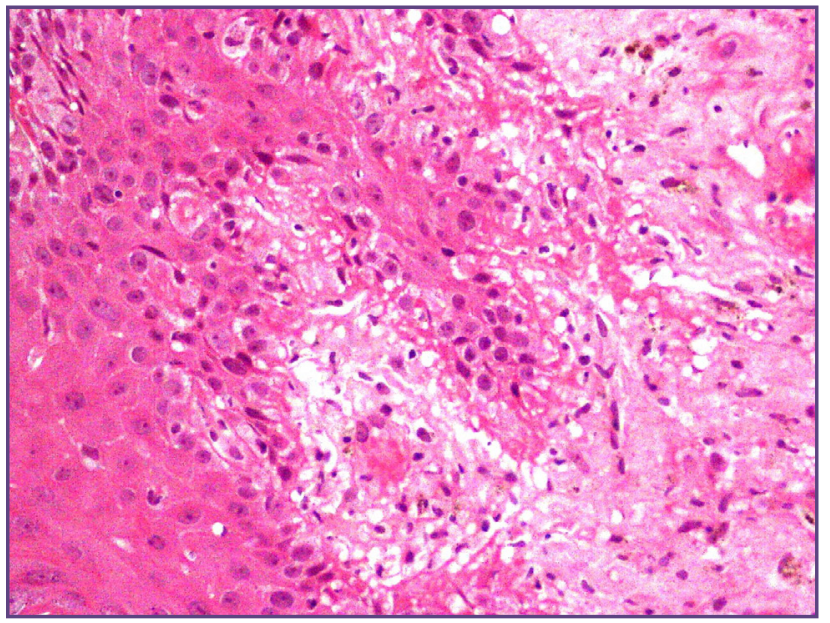

Fig. 3: Higher magnification (H/E, $x$ 400) showing lypmphocytic exocytosis causing basal cell degeneration along with presence of few apoptotic keratinocytes. In addition there is mucinous degeneration of the dermis.

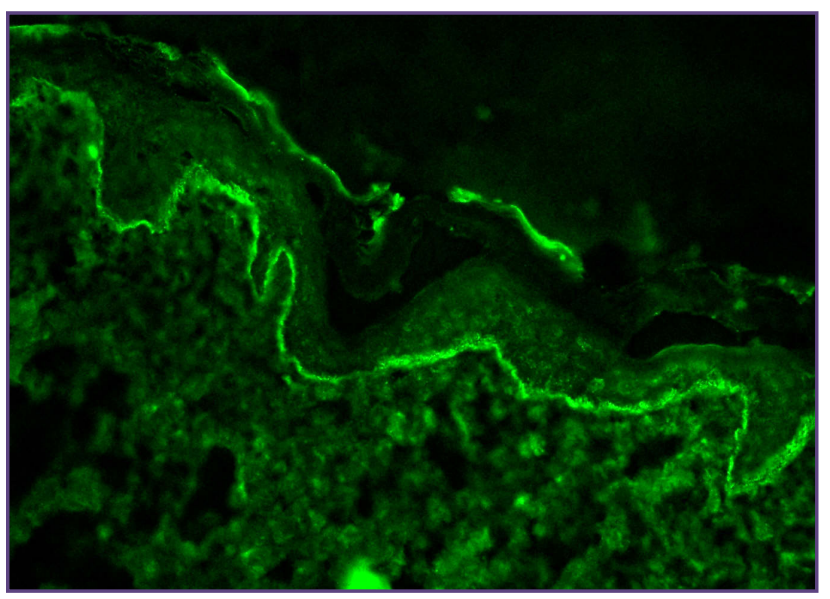

Fig. 5: DIF showing lupus band test.

Environmental triggers like exposure to ultraviolet light, drugs like TNF- $\alpha$ antagonists may unmask the disease in predisposed individuals. Clinical presentation is not dissimilar to those in adults with lesions usually localized to the face and the scalp, showing characteristic follicular plugging, and scaling, and healing with scarring and atrophy. Linear lesions that follow the lines of Blaschko have also been described. ${ }^{[4]}$ If untreated, discoid lesions may heal with hyperpigmentation or hypopigmentation and atrophy. Mucosal membrane lesions (gingivitis, mucosal hemorrhage, erosions, and small ulcerations) are seen in $3 \%$ of patients with cutaneous LE.

Photosensitivity may be present in $36 \%-81 \%$ of patients. ${ }^{[2]}$ The association with SLE is seen in $23.5 \%$ of patients, 
a proportion higher than that observed in adult DLE. ${ }^{[3]}$ Disseminated lesions are much more frequent in patients who fulfill the criteria for SLE (87.5\% vs 34\%) and hence may be a marker of poor prognosis. In $24-26 \%$ patients, childhood DLE may progress to SLE. ${ }^{[5,6]}$ In $75 \%$ of the patients of pediatric SLE, the DLE lesions are present at the onset of systemic signs, but such children may fulfill the criteria for SLE much later; thus requiring a more prolonged follow up. Arthralgia and Raynaud's phenomenon are the most frequent systemic findings in pediatric DLE and inflammatory arthritis may herald progression to SLE. ${ }^{[2]}$ The confined or disseminated character of the lesions, early onset of the disease (age of less than 10 years), disease duration, presence of antinuclear antibodies, and poor response to antimalarials are not predictive factors for progression to SLE. Antinuclear antibodies are positive in $19.2 \%$ children with DLE without SLE and in $87.5 \%$ with DLE and SLE. Histology is similar to that in adults except that epidermal atrophy is less common compared to those in adults and dermis shows a diffuse dermal infiltrate

Management of pediatric DLE involves strict photo protection, use of superpotent topical steroids, calcineurin inhibitors (tacrolimus, pimecrolimus) and oral antimalarials like hydroxychloroquin (4-6 mg/kg/d). Prednisolone in doses of 1 to $3 \mathrm{mg} / \mathrm{kg} /$ day tapered over a 2 -week period can be prescribed in conjunction with antimalarial agents to rapidly control the disease. There is no evidence suggesting that children have an increased incidence of subacute and chronic toxicity for antimalarials. Fatal reactions have been limited only to accidental or intentional overdosage.

\section{Conclusion}

DLE in our set up is rare in children. There are more chances of having a positive family history. Considering that lupus erythematosus is not a static disease and progression from DLE to SLE is much higher than in adults, patients should be continually followed up and therapy modified in response to the patient's clinical course and disease activity.

\section{References}

1. Burch PR, Rowell NR. The sex- and age-distributions of chronic discoid lupus erythematosus in four countries. Possible aetiological and pathogenetic significance. Acta Derm Venereol. 1968; 48: 33-46.

2. Moises-Alfaro C, Berrón-Pérez R, Carrasco-Daza D, Gutiérrez-Castrellón P, Ruiz-Maldonado R. Discoid lupus erythematosus in children: clinical, histopathologic, and follow-up features in 27 cases. Pediatr Dermatol. 2003;20:103-7.

3. Sampaio MC, de Oliveira ZN, Machado MC, dos Reis VM, Vilela MA. Discoid lupus erythematosus in children-a retrospective study of 34 patients. Pediatr Dermatol. 2008;25:163-7.

4. Magana M, Vazquez R. Discoid lupus erythematosus in childhood. Pediatr Dermatol 2000;17:241-2.

5. Van Gysel D, de Waard-van der Spek FB, Oranje AP. Childhood discoid lupus erythematosus: report of five new cases and review of the literature. J Eur Acad Dermatol Venereol. 2002;16:143-7.

6. Arkin LM et al. The natural history of pediatric-onset discoid lupus erythematosus. J Am Acad Dermatol. 2015;72(4):628-33.

*Corresponding author:

Poonam Adlakha, 648/30 Adarsh Nagar, Rohtak, India

Phone: +91 8813845435

Email: poonam945@yahoo.com

Date of Submission : 24.09.2016

Date of Acceptance : 19.12.2016

Financial or other Competing Interests: None.
Date of Publication : 19.02.2017 